\title{
Automatic hybrid mesh generation for the boundary face method
}

\author{
Cheng Huang \& Jianming Zhang \\ State Key Laboratory of Advanced Design and Manufacturing \\ for Vehicle Body, Hunan University, China
}

\begin{abstract}
A new hybrid mesh generation method is proposed and applied to generate mesh for three-dimensional solids with open-ended tubular holes. Regions around the tubular holes are represented by hexahedral elements, and tetrahedral elements tessellate the rest of the domain. The procedure consists of three steps. Firstly, surfaces of tubular holes are recognized and discretized, and then the hexahedral mesh is generated by a sweep method. Finally, the Delaunay tetrahedral mesh generation method coupled with the advancing front method is applied to generate tetrahedral mesh. By the proposed method, high-quality hybrid mesh for solids with small tubular holes can be automatically created. And the number of the obtained elements is far less than the traditional tetrahedral mesh. Several examples are presented to demonstrate the robustness and efficiency of our method.
\end{abstract}

Keywords: hybrid mesh, sweeping, Delaunay method, advancing front method.

\section{Introduction}

Structures containing many small holes find important application in engineering, such as cooling passages, electrical wiring passages, etc. For instance, in construction process of concrete dams many slender holes are designed as cooling passages. These pipes have significant influences on the resulting temperature and flux distribution in a body. Thus the design engineers draw much attention to properly arrange these pipes.

The analysis of solids with small tubular holes is still a difficult task by finite element method (FEM), as the FEM requires a very fine mesh to make sure the accuracy of the result. Usually, a very large amount of modeling efforts are 
necessary for mesh generation in a body with many small holes, especially when the holes are very small in size. The boundary face method (BFM) [1] has been introduced to analyses steady-state heat conduction problem [2] and elastic problem [3] of three-dimensional solids with small tubular shaped holes of free shape. The BFM is implemented directly based on the boundary representation data structure (B-rep) that is used in most CAD packages for geometry modeling. Each bounding surface of geometry model is represented as parametric form by the geometric map between the parametric space and the physical space. Both boundary integration and variable approximation are performed in the parametric space. The integrand quantities are calculated directly from the faces rather than from elements, and thus no geometric error will be introduced [4]. Usually, the boundary element method only needs to mesh the surface of the domain. The volume mesh is generated for boundary face method. The volume mesh is used to apply volume integration for the boundary face method and also be used to obtain and display the results of the arbitrary internal points.

Mesh quality has a large impact on solution quality of the CAE analysis. A high quality mesh increases the accuracy of the CAE solution. The number of elements has a large impact on the data and time costs for CAE analysis. For meshing based on complex geometry, hybrid meshes has the high mesh quality strategy and contains fewer elements than all-tetrahedral mesh. The hybrid mesh generation method can generate appropriate elements for different regions. A number of hybrid mesh generation methods have been developed and implemented for complex geometries [5-9]. This paper presents a threedimensional hybrid mesh generation method to discretize three-dimensional solids with open-ended tubular holes. In our method, the region around these tubular holes is represented by hexahedral elements and the rest of the region with complex geometry is described by tetrahedral elements. This work is a part of the framework of boundary face method. In our method, the hybrid meshes have the ease of tetrahedral meshing on complex geometries with the advantages of hexahedral cells in regions around the holes. The stress concentration always appears near the tubular holes. Results from a hexahedral mesh are more accurate than a tetrahedral mesh. Thus, hybrid mesh is very suitable for the solids with tubular holes. Compared to all-tetrahedral meshes, our method can generate far fewer elements within a reasonable time limit, resulting in substantial savings in both data preparation and computing costs. And our method can generate mesh for structures containing holes of arbitrary shape.

In the following sections, section 2 describes the general scheme of the mesh generation algorithm. Hexahedral mesh generation process is described in section 3. In section 4, the tetrahedral mesh generation method is discussed. Several examples are provided in section 5 . And the paper ends with conclusions in section 6.

\section{Scheme of the method}

The hybrid mesh generation process consists of three steps. Firstly, surfaces of tubular holes are recognized and discretized, then generating the hexahedral 
mesh around tubular holes. Finally, the tetrahedral mesh in the rest of the region is generated. While the tetrahedral mesh generation is a well-known process, the construction of the hexahedral mesh around tubular holes constitutes a major issue.

The scheme of the hybrid mesh generation method is described in Figure 1. Let's take a cube with a tubular hole for example. The hexahedral mesh generation process starts from the recognition of surfaces of tubular holes. Then the central line of the tubular hole is discretized. The hexahedral mesh is generated by sweep method along the central line of the tubular hole, as shown in Figure 1(a). After hexahedral mesh generation, the rest of the region is discretized into tetrahedral elements. At first, edges of the domain are discretized, and new fronts are added into the initial front array. Then surface triangular mesh is generated by advancing front method, as shown in Figure 1(b). And the quadrangle elements of the hexahedral mesh are discretized into several triangular elements, as shown in Figure 1(c). These triangular elements are set as surface mesh, and then the Delaunay coupled with advancing front method is used to generate the tetrahedral mesh. Then, we get the final hybrid mesh, as shown in Figure 1(d).

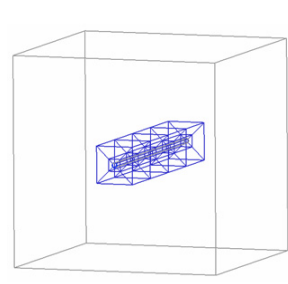

(a)

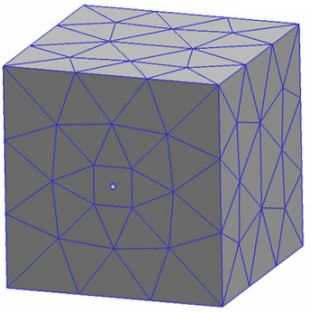

(b)

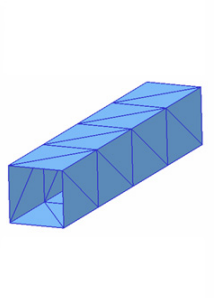

(c)

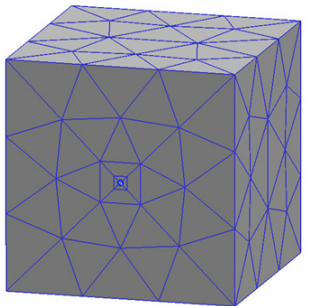

(d)

Figure 1: $\quad$ The scheme of the hybrid mesh generation method.

The boundary face method is different from the finite element method, because there is no need of a global conforming mesh for the volume integration, obtaining and displaying the results of the arbitrary internal points. Thus, there is no need of a transition mesh between hexahedral mesh and tetrahedral mesh. For generating a global conforming mesh, the pyramidal element can be used as the transition element connecting together the hexahedral mesh and tetrahedral mesh [10].

Each of these steps is briefly described in the following sections. First, the hexahedral mesh generation is detailed step by step. Afterwards, the tetrahedral mesh generation is described.

\section{Hexahedral mesh generation}

\subsection{The recognition of the surface of the tubular hole}

At first, surfaces of tubular holes need to be recognized from the geometry model. The BFM is implemented directly based on the boundary representation 
data structure (B-rep) that is used in most CAD packages for geometry modelling. In the B-rep scheme, a solid is modelled by its boundary faces which are bounded regions on planes or surfaces. Without concise mathematical formula for its explicit representation, such a boundary face is described by its bounding loops, edges and vertices [11]. In this work, the rule-based feature recognition method is used to recognize the surface of the tubular hole. In this approach, a set of heuristic rules are used to describe the operational definition for a class of features, which are similar in shape. In this work, reasonable rules are proposed to ensure the robustness of the feature recognition algorithm.

In order to recognize the solid containing open-ended tubular holes, we need to determine if the solid contains surfaces of tubular holes. The surface of the tubular hole is a closed surface. It is similar to a cylinder surface. Sometimes, the surface of the tubular hole may be a trimmed surface. The surface of an openended tubular hole intersects one or two external surfaces of the solid. The diameter of the hole is a very small value compared with the side length of the solid. A definition of a class of the surface of the open-ended tubular holes is quoted below.

- The surface of a tubular hole is a closed surface in the parametric space.

- The surface of a tubular hole contains two loops $L_{1}$ and $L_{2}$.

- $L_{1}$ contains only one edge $E_{1} . E_{1}$ is a closed curve.

- $L_{2}$ contains only one edge $E_{2} . E_{2}$ is a closed curve.

- $E_{1}$ is an inner loop of another surface $F_{\mathrm{n}} \cdot l_{\text {avg }}$ is the average length of edges contained in the outer loop of $F_{\mathrm{n}}$. The length $l_{1}$ of $E_{1}<0.2 l_{\text {avg }}$.

- $\quad E_{2}$ is an inner loop of another surface $F_{\mathrm{m}} \cdot l_{\text {avg }}$ is the average length of edges contained in the outer loop of $F_{\mathrm{m}}$. The length $l_{2}$ of $E_{2}<0.2 l_{\text {avg. }} . F_{\mathrm{n}}$ and $F_{\mathrm{m}}$ may be the same surface.

Loop over the blocks of a dam. If one surface of a solid satisfies these above constraints, the solid is recognized as the blocks with tubular holes.

\subsection{The discretization of the surface of the tubular hole}

The discretization of the surface of the tubular hole contains two parts. One is the discretization along the circumferential direction of the tubular hole; the other is the discretization along the axial direction of the tubular hole. Assume that $u$ is the axial direction and $v$ is the circumferential direction of the surface $f(u, v)$ in the parametric space. The surface is periodic in the circumferential direction.

The discretization along the circumferential direction is the discretization of two edges of the surface of the tubular hole. The surface of the tubular hole is a closed surface. For a closed surface, the boundary polygon is not closed in the parametric space [12]. These two edges are periodic parametric curves. At first, one edge is discretized. Then, the first discretization point of the other edge needs to be located. In order to make sure these two first points of two edges are in the same period, and these discretization points of two edges are consistent. 
The aim of the discretization along the axial direction is to obtain the discretization of the central line of the tubular hole. However, there is not a central line of the tubular hole in the geometry model. We select two parallel curves of the surface. And discretize these two curves. The middle points of the corresponding two points of these two curves are calculated. These middle points are the discretization of the central line of the tubular hole. Let $u=0$ and $u=0.5$ are two curves of the surface $f(u, v)$ in the parametric space. These two curves are discretized by the parametric curve discretization algorithm with the same rule. In this work, the algorithm according to the arc length rule for the discretization of 3D parametric curve is applied [13]. Appling this process, finally we can get the discretization of the curve.

\subsection{Sweep method for hexahedral mesh generation}

Hexahedral elements are generated by the sweep method around the tubular holes. Sweeping is a method of meshing two and one half dimensional volumes with an all hexahedral mesh [14-16]. A two and one half dimensional volume is a volume that has a topologically constant cross section along a single axis known as the "sweep axis." These volumes can be defined by a source surface, a target surface and a series of link surface. The source and target surfaces may have different areas and curvatures. However, they must be topologically equivalent. Thus, they must have the same number of holes and logical sides. Furthermore, link surfaces have to be mappable. Hence, they must have only four logical sides [14].

In this work, a predigest sweep method is applied to generate hexahedral mesh. At first, a quadrilateral mesh is generated over the source surface. Then, the target surface mesh is generated. After that, the inner layers of nodes and elements and the structured quadrilateral mesh over the linking-sides are generated at the same time.

Firstly, a quadrilateral mesh is generated over the source surface. In a solid contains tubular holes, there is one or two surface intersected by a tubular hole. One surface is set as source surface; the other is set as target surface. If a tubular hole intersects one surface twice, the source surface and target surface will be the same surface. Not the whole source surface is discretized, only the region of the surface around the hole is discretized into quadrilateral mesh. It is a simple quadrangular mesh. According to the number of segments along circumferential direction of the hole and the number of layer of the quadrangular mesh, the quadrangular mesh around the hole is created. This quadrangular mesh is set as source surface mesh, and the sweep method is applied to generate hexahedral mesh along the axial direction of the tubular hole.

The discretization of the central line of the tubular hole has been obtained in the previous step. The number of segments of the central line is the number of layers of the hexahedral elements. For every layer, there is one outer loop, and one inner loop for each hole in the sweep volume. The discretization of the out loop and inner loop of each layer can be easily gotten. Then, the following algorithm is used to generate hexahedral mesh along the central line of the tubular hole. 
After the quadrangle mesh generation of the source surface, the quadrangle mesh is projected onto the target surface. The projection method between parametric surfaces and the least-squares approximation are applied to obtain the target surface mesh. The mapping is defined between the parametric spaces of these two surfaces. Thus, this sweep algorithm can be applied when the source surface and target surface have different curvatures. Our program is implemented directly based on the boundary representation data structure (B-rep). Each bounding surface of geometry model is represented as parametric form by the geometric map between the parametric space and the physical space. The parametric coordinates of nodes of the source and target surfaces can be easily obtained. Then, the rest of the boundary nodes and inner nodes of the extrusion volume have to be generated. These nodes have to be placed, layer by layer, along the axial direction of the tubular hole. The details of the projection method can be found in references [14]. After the boundary and inner nodes of $k$-th level are generated, the respect hexahedral elements and the structured quadrilateral mesh over the link surfaces can be easily generated by joining the corresponding nodes between adjacent layers.

\section{Tetrahedral mesh generation}

The process of the tetrahedral mesh generation contains three steps. At first, all edges are discretized. These segments of discretized edges are set as initial front for triangular mesh generation. And the outside edges of quadrangular meshes in the source and target surface are also set as initial front. We have developed an adaptive triangular mesh generation method over an arbitrarily parametric surface based on the advancing front method combined with a quad-tree procedure [4]. Then, this advancing front method is used to generate the surface triangular mesh, as shown in Figure 1(b). After that, outside quadrangular cells of the hexahedral mesh are discretized into two triangular cells, as shown in Figure 1(c). These triangular cells are set as surface triangular mesh. Finally the Delaunay tetrahedral mesh generation method coupled with the advancing front method is used to generate the tetrahedral mesh of the rest of the region [17].

The quality and efficient Delaunay tetrahedral mesh generation method coupled with the advancing front method is applied to generate tetrahedral mesh. The combined method has the high quality point placement strategy of the advancing front method and the high efficient and the mathematical properties of the Delaunay approach. We suggest using the advancing front method as an internal point creation tool, the Delaunay algorithm as an insertion tool and the Delaunay boundary tetrahedral mesh as a background mesh. Similar to the conventional advancing front method, the front is identified and internal points are created to form optimal tetrahedral elements in accordance with an elementsize defined by the control space. A Delaunay kernel procedure is used to insert these points into the existing triangulation. Thus the convergence of the process is always ensured.

First step of the tetrahedral mesh generation algorithm is boundary tetrahedral mesh generation. At first, we construct a bounding box enclosing the domain. 
The box as a convex environment encloses all the boundary points. The box is defined by eight vertices and it is divided into five tetrahedrons. All the boundary points are inserted into the initial mesh using the Delaunay kernel. Then, the boundary is recovered, and we obtain the boundary tetrahedral mesh.

After the boundary tetrahedronization, the domain tetrahedral mesh is generated. The tetrahedral mesh is obtained by adding field points inside the existing mesh and then optimized so as to complete the final mesh of the domain. Similar to the conventional advancing front method, we define the suitable triangle generated in the last step as the front. And all the triangles of the boundary triangular discretization are defined as the initial fronts. We define the front which can be used to create new points as the active front. The internal points are iteratively created by the active front to form optimal tetrahedral elements and inserted into the previous mesh by Delaunay kernel. The quality of the generated mesh can be improved dramatically by several techniques of mesh optimization. So, after the mesh generation the Laplacian smoothing and topological optimization process are used to improve the quality of the mesh. This method can generate high quality tetrahedral mesh within a reasonable time limit.

\section{Examples of application}

In this section, several hybrid meshes are presented to demonstrate the robustness and efficiency of the proposed method. All the mesh generation process are carried out on the desktop computer with Intel (R) Core(TM)2 Duo CPU E7400 (2.80GHz).

\subsection{Block with a hole}

The first example is a block with a curved cylindrical hole. It is an $8 \times 10 \times 4$ block with an open-ended cylindrical hole as shown in Figure 2(a). The diameter of the cooling passage is 0.2 . It is discretized by the proposed method. The mesh consists of 336 hexahedral elements, 3073 tetrahedral elements, and 1177 mesh nodes. The total CPU time needed for the mesh generation is less than 1 seconds. The final hybrid mesh is depicted in Figure 2(b).

For tetrahedral mesh, the shape metric, $Q_{T}$, is the ratio of respective radii of the inscribed to circumscribed spheres of the tetrahedron. For tetrahedral elements, the element shape metric approaches 1.0 for a perfect element, and approaches 0.0 for degenerated element of zero volume. The Jacobian determinant $J_{m}$ and condition number Cond are used as the mesh quality estimate criteria of hexahedral element [18]. When $0.5 \leq J_{m} \leq 1.0$ and $1 \leq$ Cond $\leq 4$, the hexahedral element quality is acceptable for numerical analysis. The element shape metrics of the hybrid mesh is listed in Table 1. In this case, our method can generate high quality hybrid mesh.

The mesh generated by our method is compared to the mesh generated by Hypermesh. Hypermesh is a powerful meshing software. The resulting mesh is all tetrahedral mesh, because this solid with a curved hole is hard to be 
discretized into all hexahedral mesh automatically. Figure 3 shows the mesh generated by Hypermesh. We can see that a very fine mesh is generated around the tubular hole. The mesh generated by Hypermesh contains 6990 mesh nodes, 36027 tetrahedral elements. We can see that the number of tetrahedral elements generated by Hypermesh is almost 10 times as large as the number of hybrid elements generated by our method. Compared to traditional tetrahedral mesh, our method can generate hybrid mesh with fewer elements, resulting in substantial savings in both data preparation and computing costs.

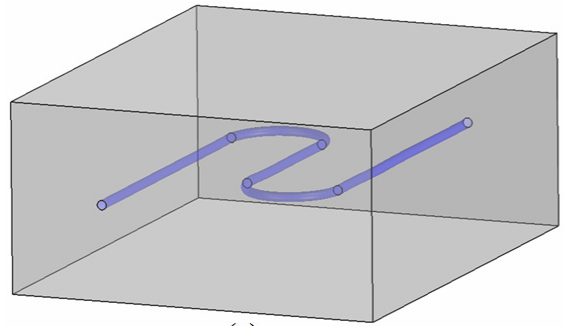

(a)

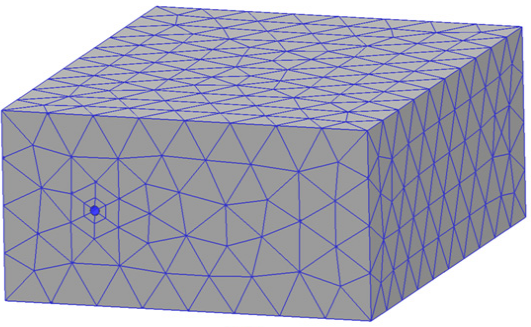

(b)

Figure 2: (a) Block with a hole and (b) the hybrid mesh.

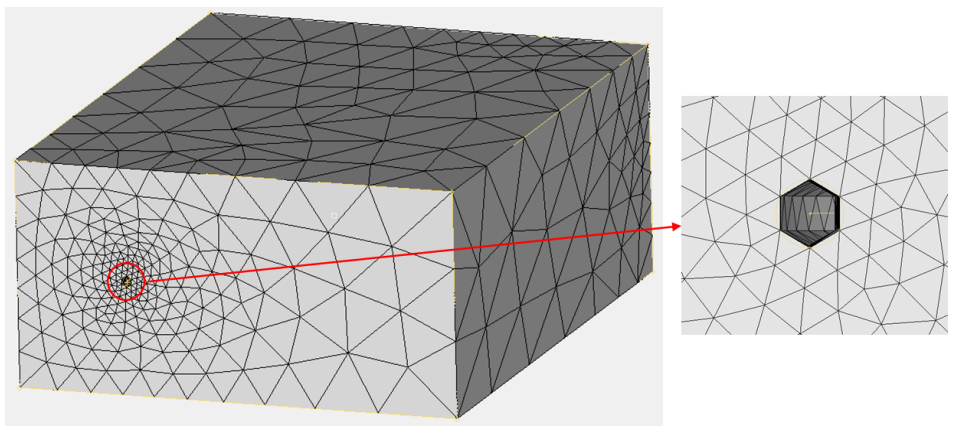

Figure 3: The mesh generated by hypermesh.

Table 1: $\quad$ Mesh results.

\begin{tabular}{|c|c|c|c|c|}
\hline \multicolumn{2}{|c|}{} & Example 1 & Example 2 & Example 3 \\
\hline \multirow{4}{*}{$\begin{array}{c}\text { Tetrahedral } \\
\text { mesh }\end{array}$} & Num. element & 3073 & 5384 & 3077 \\
\cline { 2 - 5 } & CPU Time & $<1 \mathrm{~s}$ & $6 \mathrm{~s}$ & $1 \mathrm{~s}$ \\
\cline { 2 - 5 } & Min. metric & 0.351568 & 0.305876 & 0.258136 \\
\cline { 2 - 5 } & Avg. metric & 0.857233 & 0.766175 & 0.802674 \\
\hline \multirow{4}{*}{$\begin{array}{c}\text { Hexahedral } \\
\text { mesh }\end{array}$} & Num. element & 336 & 480 & 44 \\
\cline { 2 - 5 } & CPU Time & $<1 \mathrm{~s}$ & $<1 \mathrm{~s}$ & $<1 \mathrm{~s}$ \\
\cline { 2 - 5 } & Min. $J_{m}$ & 0.675013 & 0.657904 & 0.501374 \\
\cline { 2 - 5 } & Avg. $J_{m}$ & 0.705291 & 0.703524 & 0.695653 \\
\cline { 2 - 5 } & Min. Cond & 1.423571 & 1.376605 & 1.527384 \\
\cline { 2 - 5 } & Avg. Cond & 1.451309 & 1.447680 & 1.769102 \\
\hline
\end{tabular}


Then, the diameter of the hole is specified as a smaller value 0.1 . The hybrid mesh generated by our method is the same as the previous case, while the mesh generated by Hypermesh contains 17207 mesh nodes and 92009 tetrahedral elements. The smaller the hole is, the more obvious the advantage of our method is.

\subsection{Block with sever holes of arbitrary shape}

The second example is a $10 \times 10 \times 10$ block with 4 open-ended cylindrical holes of arbitrary shape, as shown in Figure 4(a). The diameter of the holes is 0.2. This example is more geometrically complicated. It is considered here to show the advantage of our method to generate mesh for structures containing many openended tubular holes of arbitrary shape. Mesh generated by our method just contains 480 hexahedral elements, 5384 tetrahedral elements, and 1779 mesh nodes. The total CPU time needed for the mesh generation is 6 seconds. A view of the hexahedral mesh around one curve hole is depicted in Figure 5. The final hybrid mesh is depicted in Figure 4(b). Table 1 shows the element shape metrics of the hybrid mesh. In this case, our method also can generate high quality mesh over the rest of the region; even the block contains 4 open-ended cylindrical holes of arbitrary shape.

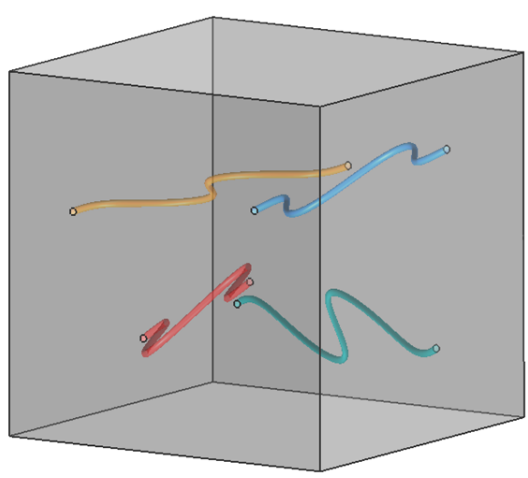

(a)

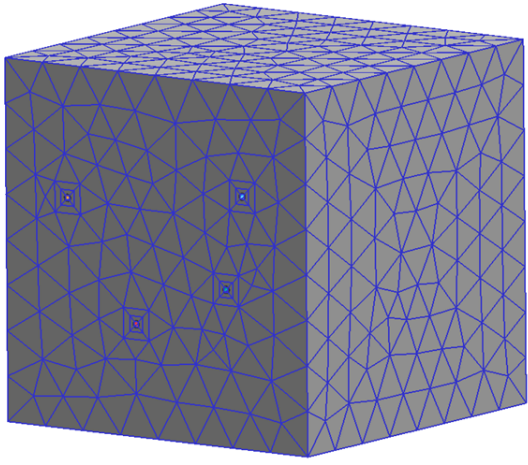

(b)

Figure 4: (a) A block with 4 open-ended cylindrical holes of arbitrary shape and (b) the hybrid mesh.

\subsection{Block of a practical concrete dam}

The fourth example is a part of the large crankshaft used by ship's diesel engine, as shown in Figure 5(a). This crankshaft is much bigger than the ordinary crankshaft. The diameter of the main journal is 295 . The diameter of the connecting rod journal is 290 . The length of the whole crankshaft is 49625 . However, the diameter of the oilhole is 12 , a very small value. The stress concentration always appears near the oilhole, so it cannot be ignored during the CAE analysis. Mesh generated by the presented method contains 44 hexahedral 
elements, 3077 tetrahedral elements, and 838 mesh nodes. The total CPU time needed for the mesh generation is 1 seconds. A view of the tetrahedral mesh over the rest of the region is depicted in Figure 5(b). The final hybrid mesh is depicted in Figure 6(a). Table 1 shows the element shape metrics of the hybrid mesh. We can see that our method can generate high quality tetrahedral mesh for complex geometry.

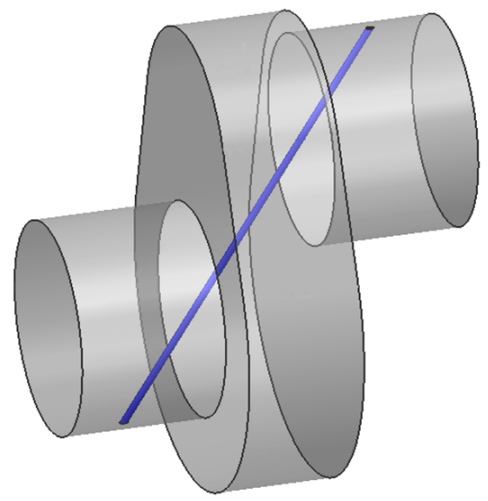

(a)

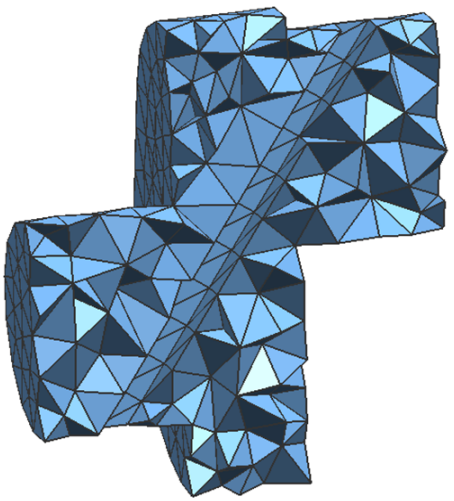

(b)

Figure 5: (a) A part of the large crankshaft used by ship's diesel engine and (b) the tetrahedral mesh over the rest of the region.

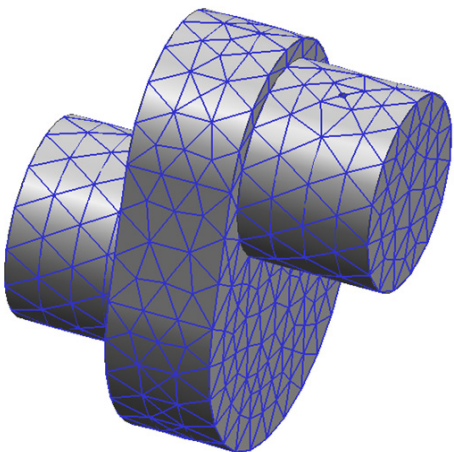

(a)

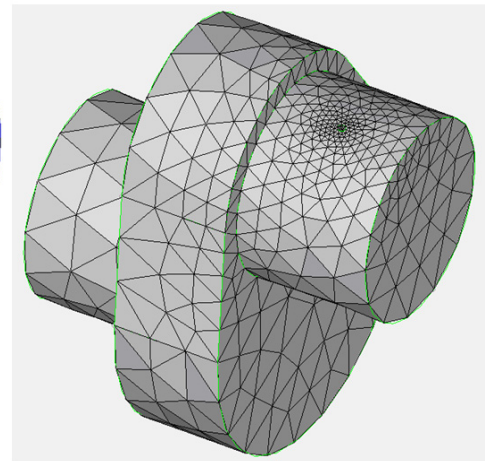

(b)

Figure 6: (a) The hybrid mesh and (b) the mesh generated by Hypermesh.

Figure 6(b) shows the mesh generated by Hypermesh. The mesh generated by Hypermesh contains 4321 mesh nodes and 21496 tetrahedral elements. The total CPU time needed for the mesh generation is 1 seconds. The number of tetrahedral elements generated by Hypermesh is almost 7 times as large as the number of hybrid elements generated by our method. Thus, the present method is applicable for generating hybrid mesh for complex solid with small tubular holes. 


\section{Conclusions}

A hybrid mesh generation method for three-dimensional solids with open-ended tubular holes has been presented in this paper. The algorithm according to the arc length rule for the discretization of parametric curve has been applied to obtain the discretization of the central line of the tubular hole. The sweep method has been applied to generate hexahedral elements around these tubular holes. The Delaunay tetrahedral mesh generation method coupled with the advancing front method has been applied to generated tetrahedral mesh of the rest of the region. Thus the discretization of the domain is much simpler than the conventional tetrahedral mesh generation method. And the number of hybrid elements generated by our method is far less than the traditional tetrahedral mesh. Therefore, the amount of data and computational cost are considerably reduced. The robustness and efficiency of the proposed method have been verified through several examples.

\section{Acknowledgement}

This work was supported by the National Science Foundation of China under grant number 11172098 .

\section{References}

[1] Zhang JM, Qin XY, Han X and Li GY. A boundary face method for potential problems in three dimensions. International Journal for Numerical Methods in Engineering 2009; 80(3): 320-337.

[2] Qin XY, Zhang JM, Liu LP and Li GY. Steady-state heat conduction analysis of solids with small open-ended tubular holes by BFM. International Journal of Heat and Mass Transfer 2012.

[3] Huang C, Zhang JM, Qin XY, Lu CJ, Sheng XM and Li GY. Stress analysis of solids with open-ended tubular holes by BFM. Engineering Analysis with Boundary Elements 2012; 36(12): 1908-1916.

[4] Qin XY, Zhang JM, Li GY, Sheng XM, Song Q and Mu DH. An element implementation of the boundary face method for 3D potential problems. Engineering Analysis with Boundary Elements 2010; 34: 934-943.

[5] Kallinderis Y, Khawaja A, McMorris H. Hybrid prismatic/tetrahedral grid generation for viscous flows around complex geometries. AIAA journal 1996; 34(2): 291-298.

[6] Athanasiadis AN, Deconinck H. Object-oriented three-dimensional hybrid grid generation. International Journal for Numerical Methods in Engineering 2003; 58(2): 301-318.

[7] Chand KK. Component-based hybrid mesh generation. International Journal for Numerical Methods in Engineering 2005; 62(6): 747-773.

[8] Flandrin N, Borouchaki H, Bennis C. 3d hybrid mesh generation for reservoir flow simulation. In: Proceedings of the 13th international meshing roundtable. $2004,133-144$. 
[9] Khawaja A, Minyard T, Kallinderis Y. Adaptive hybrid grid methods. Computer Methods in Applied Mechanics and Engineering 2000; 189(4): 1231-1245.

[10] Meyers RJ, Tautges TJ, Tuchinsky PM. The 'Hex-Tet'hex-dominant meshing algorithm as implemented in CUBIT. In: Proceedings of the 7th international meshing roundtable. 1998, 151-158.

[11] $\mathrm{Wu} \mathrm{MC,} \mathrm{Lit} \mathrm{CR.} \mathrm{Analysis} \mathrm{on} \mathrm{machined} \mathrm{feature} \mathrm{recognition} \mathrm{techniques}$ based on B-rep. Computer-Aided Design 1996; 28(8): 603-616.

[12] Guan ZQ, Shan JL, Zheng Y, et al. An extended advancing front technique for closed surfaces mesh generation. International Journal for Numerical Methods in Engineering 2008; 74(4): 642-667.

[13] Cuilliere JC. An adaptive method for the automatic triangulation of 3D parametric surfaces. Computer-Aided Design 1998; 30(2): 139-149.

[14] Roca X, Sarrate J, Huerta A. Surface mesh projection for hexahedral mesh generation by sweeping. In: Proceedings of the 13th international meshing roundtable. 2004, 169-179.

[15] Knupp PM. Next-generation sweep tool: a method for generating all-hex meshes on two-and-one-half dimensional geometries. In: Proceedings of the 7th international meshing roundtable. 1998, 505-513.

[16] Staten ML, Canann SA, Owen SJ. BMSweep: locating interior nodes during sweeping. In: Proceedings of the 7 th international meshing roundtable. 1998, 7-18.

[17] Huang C, Zhang JM, Liu LP, Li GY. A quality and efficient tetrahedral mesh generation method. Australian Journal of Mechanical Engineering, accept.

[18] Knupp PM. Achieving finite element mesh quality via optimization of the Jacobian matrix norm and associated quantities. Part II- a framework for volume mesh optimization and the condition number of the Jacobian matrix. International Journal for Numerical Methods in Engineering, 2000, 48(8): 1165-1185. 